\title{
Laminar MHD Flow in the Entrance Region of an Annular Channel
}

\author{
S. Venkateswarlu ${ }^{1}$ B. Rama Bhupal Reddy ${ }^{2}$, R. Siva Prasad ${ }^{3}$ \\ ${ }^{I}$ Assoc. Prof., Dept. of Mathematics, R.G.M. Engineering College, Nandyal, A.P., India. \\ ${ }^{2}$ Assoc. Prof., Dept. of Mathematics, K.S.R.M. College of Engineering, Kadapa, A.P., India. \\ ${ }^{2}$ Professor, Dept. of Mathematics, S.K. University, Anantapur, A.P., India.
}

\begin{abstract}
In this paper we analyze the Laminar MHD flow in the entrance region of an annular channel under the influence of transverse magnetic field. The motion of the fluid is between the two insulating cylinders which are concentric. The origin of the coordinate system is located at the extreme left of the channel along the central line of the cylinders, $z$ is the coordinate which increases in the down stream direction, $r$ is the radial coordinate and $\theta$ is the angular coordinate and is perpendicular to the $(r, z)$ plane. The flow problem is described by means of partial differential equations and the solutions are obtained by using an implicit finite difference technique. The velocity, temperature and pressure profiles are obtained and their behavior is discussed computationally for different values of governing parameters like magnetic field parameter M and Prandtl number Pr.
\end{abstract}

Keywords: Laminar flow, MHD, Annular channel.

\section{Introduction}

Fluid transport systems composed of pipes or ducts have many practical applications in chemical plants and oil refineries, city water supply system and Bio-fluid mechanism which one seem to be maze of pipes. Power plants contain many pipes and ducts for transporting fluid involved in the energy-conservation process. Hartmann et al [5] determined the steady-state profile for laminar magneto-hydrodynamic flow in a plane channel and the analog to that problem in cylindrical co-ordinates was solved by Globe [4]. Uflyland [13] and Chekmarer [3] analyzed the time transient case of this problem, which implies a development time, rather than a development length. The entry problem for this case is solved by a technique similar to that of Shohet et al [11].

Abdul Maleque and Abdul Sattar [1] were studied the effects of variable properties and hall current on study MHD laminar convective fluid flow due to a porous rotating disk. MHD flow of a power-law fluid over a rotating disk studied Anderson and Korte [2]. Jain, Nourmohammadi and Roy [7] were studied the forced convective sub-cooled boiling in heated annular channels. Diffusion of Aerosols in the entrance region of a smooth cylindrical pipe studied Ingham [6]. Kumari and Nath [8] were studied Transient MHD rotating flow over a rotating sphere in the vicinity of the equator. Shah and Farnia [10] were studied flow in the entrance of annular tubes.

In this paper determines the velocity and temperature of laminar MHD flow in the entrance region of annular channel. Both the velocity and Temperature profiles are initially flat upon entering channel. The equations of this system are placed into a finite difference form and solved numerically for various values of magnetic field parameter and fixed prandtl number.

\section{Formulation Of The Problem}

The annular device is shown in figure 1. This representation is best described in cylindrical coordinates. The motion of the fluid is between the two insulating cylinders, which are concentric. The origin of the co-ordinate system is located at the extreme left of the channel along the center line of the cylinders, $\mathrm{Z}$ is the co-ordinate which increases in the down stream direction, $r$ is the radial co-ordinate and $\theta$ is the angular coordinate and is perpendicular to the $(\mathrm{r}, \mathrm{z})$ plane. Note that the origin of this co-ordinate system is not placed in the center of the region in which the fluid passes. The uniform magnetic field will be in the radial direction. The inner radius of the channel is $r_{i}$ and the outer radius is $r_{0}$. The channel is of length ' $L$ ', which must be long compared with the entry length. 


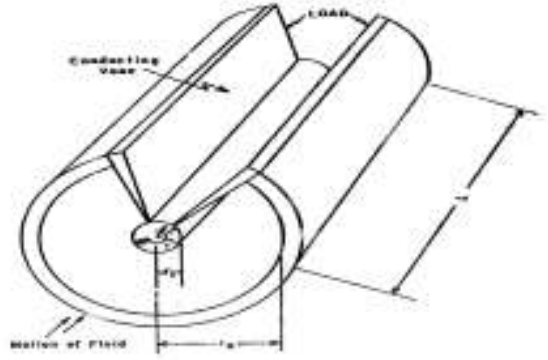

Figure 1 : Annual channel configuration

The governing equations are:

$$
\begin{aligned}
& \frac{D \rho}{D t}+=\frac{\partial \rho}{\partial \tau}+\bar{q} \cdot \nabla \rho \\
& \rho \frac{D q}{D t}=-\nabla p+\mu \nabla^{2} \bar{q}+\mu_{e} \bar{J} \times \bar{H} \\
& \rho C_{p}[\bar{q} \cdot \nabla] T=\kappa \nabla^{2} T+\phi
\end{aligned}
$$

Where $\phi=$ Dissipation function

$$
=\mu\left(\frac{\partial v_{r}}{\partial z}+\frac{\partial v_{z}}{\partial r}\right)^{2}+2 \mu\left[\left(\frac{\partial v_{r}}{\partial r}\right)^{2}+\left(\frac{\partial v_{z}}{\partial z}\right)^{2}\right]
$$

The Ohm's law is $\bar{J}=\sigma\left[\bar{E}+\mu_{e} \bar{q} \times \bar{H}\right]$

Where $\bar{q}=\left(v_{z}, v_{r}, 0\right)$ and $\bar{H}=\left(0, H_{0}, 0\right)$

The following assumptions are made:

(i) Flow is steady, laminar, viscous, incompressible and developed.

(ii) There are no applied (external) magnetic fields other than in the r-direction.

(iii) Electric field $\bar{E}$ and induced magnetic field are neglected $[9,12]$

(iv) All the physical properties of the fluid are assumed to be constant

(v) Energy dissipation is neglected.

The resultant equations in cylindrical coordinates from the above assumptions are listed below:

$$
\begin{aligned}
& \frac{1}{r} \frac{\partial\left(r u_{r}\right)}{\partial r}+\frac{\partial u_{z}}{\partial z}=0 \\
& \rho\left[u_{r} \frac{\partial u_{z}}{\partial r}+u_{z} \frac{\partial u_{z}}{\partial z}\right]=-\frac{\partial p}{\partial z}+\mu\left[\frac{\partial^{2} u_{z}}{\partial r^{2}}+\frac{1}{r} \frac{\partial u_{z}}{\partial r}\right]-\frac{\sigma \mu_{e}^{2} H_{0}^{2}}{r^{2}} u_{z} \\
& \rho C_{P}\left[u_{r} \frac{\partial t}{\partial r}+u_{z} \frac{\partial t}{\partial z}\right]=\kappa\left[\frac{\partial^{2} t}{\partial r^{2}}+\frac{1}{r} \frac{\partial t}{\partial r}\right]
\end{aligned}
$$

The boundary conditions are

$$
\begin{aligned}
& \mathrm{u}_{\mathrm{z}}=\mathrm{u}_{0}, \mathrm{u}_{\mathrm{r}}=0, \mathrm{~T}=\mathrm{T}_{\text {in }} \text { for } \mathrm{z}=0 \text { \& } \mathrm{r}_{\mathrm{i}} \leq \mathrm{r} \leq \mathrm{r}_{\mathrm{o}} \\
& \mathrm{u}_{\mathrm{r}}=0, \quad \text { for } \mathrm{z}>0 \text { and } \mathrm{r}=\mathrm{r}_{\mathrm{i}} \\
& \mathrm{u}_{\mathrm{z}}=0, \mathrm{u}_{\mathrm{r}}=0, \mathrm{~T}=\mathrm{T}_{\mathrm{w}} \text { for } \mathrm{z}>0 \text { and } \mathrm{r}=\mathrm{r}_{\mathrm{o}}
\end{aligned}
$$

The equations (4) to (6) and boundary conditions (7) are put in non-dimensional form by using the following transformations

$$
\begin{array}{llll}
Z=z \frac{\mu}{r_{i}^{2} \mu_{0} \rho} & V=\frac{\rho u_{r} r_{i}}{\mu} & R=\frac{r}{r_{i}} & P=\frac{p}{\rho u_{0}^{2}} \\
\operatorname{Pr}=\frac{\mu C_{P}}{\kappa} & U=\frac{u_{z}}{u_{0}} & T=\frac{t-t_{\text {in }}}{t_{w}-t_{\text {in }}} & M^{2}=\frac{\sigma \mu_{e}^{2} H_{0}^{2}}{\mu}
\end{array}
$$


The determining equations are then:

$$
\begin{aligned}
& \frac{1}{R} \frac{\partial(V R)}{\partial R}+\frac{\partial U}{\partial Z}=0 \\
& V \frac{\partial U}{\partial R}+U \frac{\partial U}{\partial Z}=\frac{-\partial P}{\partial Z}+\frac{\partial^{2} U}{\partial R^{2}}+\frac{1}{R} \frac{\partial U}{\partial R}-\frac{M^{2} U}{R^{2}} \\
& V \frac{\partial T}{\partial R}+U \frac{\partial T}{\partial Z}=\frac{1}{\operatorname{Pr}}\left[\frac{\partial^{2} T}{\partial R^{2}}+\frac{1}{R} \frac{\partial T}{\partial R}\right]
\end{aligned}
$$

Where $M^{2}=\frac{\sigma \mu_{e}^{2} H_{0}^{2}}{\mu}$ and $\operatorname{Pr}=\frac{\mu C_{P}}{\kappa}$

The boundary conditions are

$\mathrm{U}=1, \mathrm{~V}=0, \mathrm{~T}=0$, for $\mathrm{Z}=0$ and $1 \leq \mathrm{R} \leq 3$

$\mathrm{V}=0$ for $\mathrm{Z}>0$ and $\mathrm{R}=1$,

$\mathrm{U}=0, \mathrm{~V}=0, \mathrm{~T}=1$ for $\mathrm{Z}>0$ and $\mathrm{R}=3$

A finite difference technique is adopted for solving above differential equations (9) to (11) together with boundary conditions (12).

\section{Results And Discussion}

The following initial values are taken at entrance:

$\mathrm{Z}=0, \mathrm{U}=1.0 \mathrm{~V}=0, \mathrm{P}=0.1357 \& \mathrm{Pr}=0.1$. The Laminar magneto hydrodynamic $(\mathrm{MHD})$ flow is governed by the non-dimensional parameters, $\mathrm{M}$ magnetic field parameter and $\operatorname{Pr}$ Prandtl number. The velocity field, temperature distribution and pressure in the entrance region of an annular channel is evaluated analytically by implicit finite difference method with reference to variations in the governing parameters has been computationally analyzed.

We discuss the nature of the profiles for the velocity and temperature distributions for different sets of governing parameters namely M magnetic field parameter, Pr Prandtl number; R ratio of the concentric cylinders, $\mathrm{Z}$ variable parameter, $t_{W}$ wall temperature, $t_{i n}$ input temperature and $\mathrm{u}_{\mathrm{o}}$ uniform velocity, also for computational purpose. We are fixing non-dimensional pressure difference $\mathrm{P}$, non-dimensional stream wise velocity $\mathrm{U}$ as well as $\mathrm{V}$ non-dimensional transfer velocity. Fig.2 to Fig.11 representing the velocity profiles for U, Fig. 12 to Fig. 18 corresponds to the temperature distributions for T while Fig.19 to Fig. 21 corresponds to the pressure profiles for $\mathrm{P}$.

We notice that the induced magnetic field is fixed for all, but the ratio of the concentric cylinders it should be varied here. The magnitude of the velocity enhances initially and then gradually decreases with increasing $\mathrm{R}$ while fixing the magnetic parameter $\mathrm{M}$ and Prandlt number Pr. The magnitude of the velocity for $M=0$ are does not change the velocity relative for $M=2,4$ and 6 . Here we observe that the induced magnetic fields do not effect the motion at the fluid between the two insulating concentric cylinders Fig.2 to Fig.5. We observe that the profiles for different values of $\mathrm{R}$ (i.e. $\mathrm{R}=1.2,1.6$ and 1.8) the magnitude of the velocities reduces for the values of $Z$, i.e. $0 \leq Z \leq 0.05$ while enhances for the values $0.06 \leq Z \leq 0.09$ with increasing the magnetic parameter $M$ fixing the other parameter Fig.6 to Fig.8. Again we observe that the profiles for different $Z$, the magnitude of the velocity enhances and then immediately reduces with increasing $Z$ while fixing the other parameters. These profiles are shown for different values of the indeed magnetic field i.e $M=0,2,4$ the similar behavior of the velocity components is observed for M=0, 2 and 4 (Fig.9 to Fig.11).

We also notice that the temperature profiles shows that, it reduces initially and then gradually enhances with increasing the ratio of the concentric cylinders $\mathrm{R}$ while fixing the other parameters for $\mathrm{M}=0$. The similar behavior of the temperature components is observed for the magnetic parameters $M=2,4$ and 6 , Fig. 12 to Fig.15. Next we observe that the temperature profiles Fig.12 to Fig. 18 for different variations in the magnetic parameters. The temperature $T$ enhances with increasing the magnetic parameter $M$ (i.e $M=2,4,6$ ). The similar observations was obtained for the values of $(\mathrm{R}=1.2,1.6$ and 1.8) and fixing the other parameters. The magnitude of pressure component decreases for the values of $0 \leq R \leq 0.04$ and than enhances for $0.05 \leq R \leq 0.1$ with increasing the magnetic parameter $M(M=0,2,4,6)$ while fixing $Z=0.001$. Now we change $Z=0.01$ the magnitude of pressure enhances with increasing the magnetic parameter $M$ for all values of $Z$. The similar behavior is observed for the values of $\mathrm{Z}=0.1$ (Fig. 20 and Fig.21). Therefore in general the magnitude of 
Laminar MHD Flow in the Entrance Region of an Annular Channel

pressure enhances with increasing the indeed magnetic field in the radial direction throughout the annular channel.

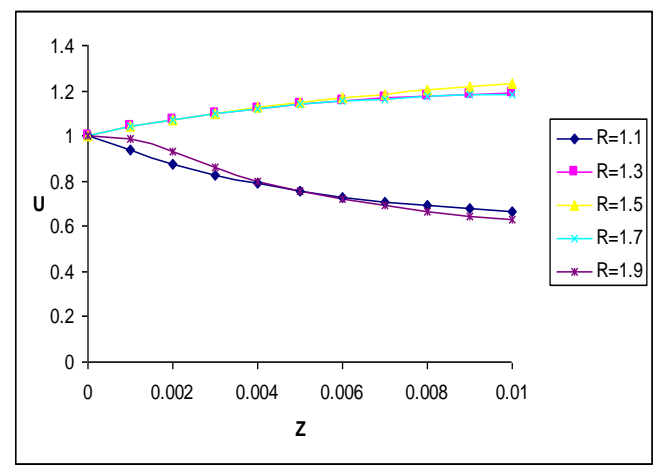

Fig 2: Velocity profile for fixed $\mathrm{M}=0$

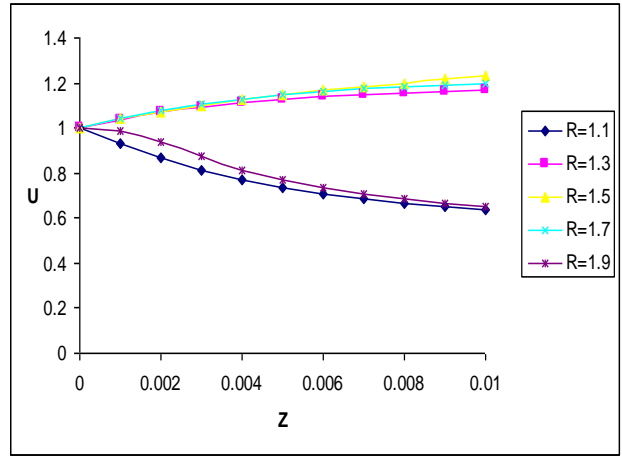

Fig.4: Velocity profile for fixed $\mathrm{M}=4$

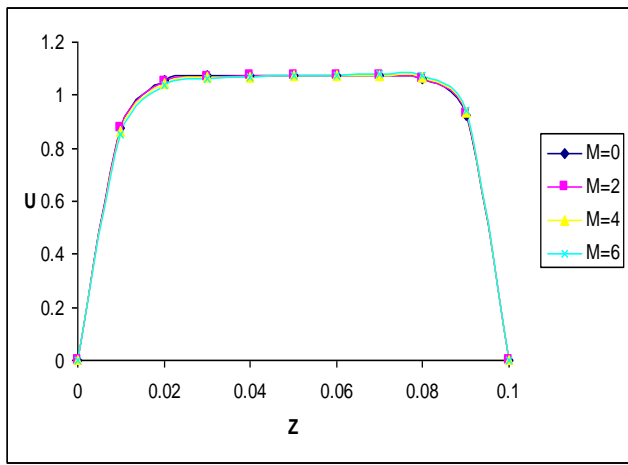

Fig.6: Velocity profile for fixed $\mathrm{R}=1$

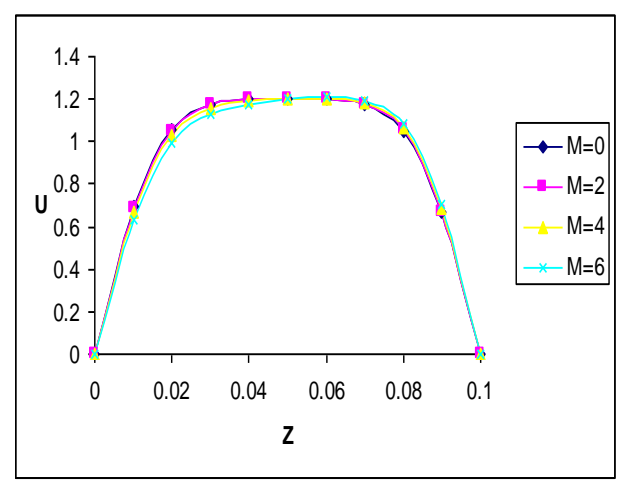

Fig.8: Velocity profile for fixed $\mathrm{R}=1.8$

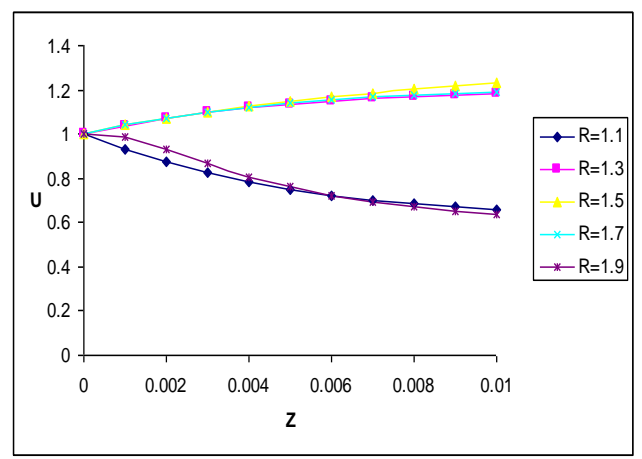

Fig.3: Velocity profile for fixed $\mathrm{M}=2$

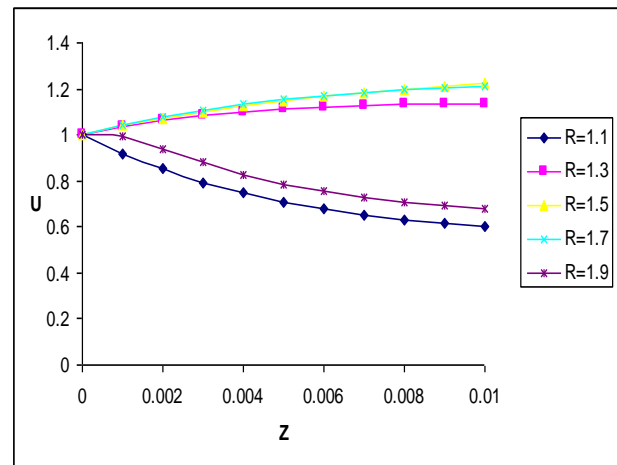

Fig.5: Velocity profile for fixed $M=6$

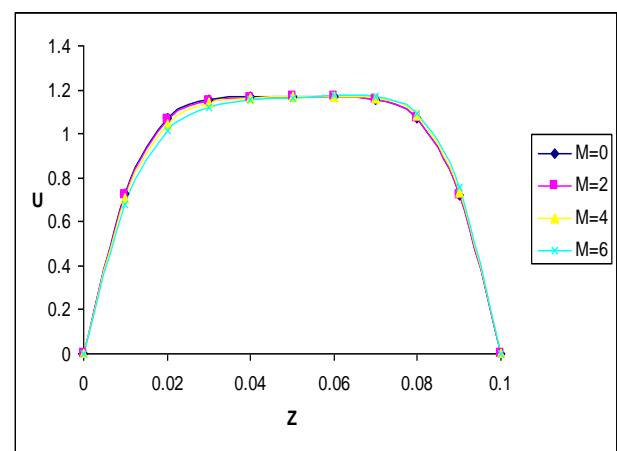

Fig.7: Velocity profile for fixed $\mathrm{R}=1.6$

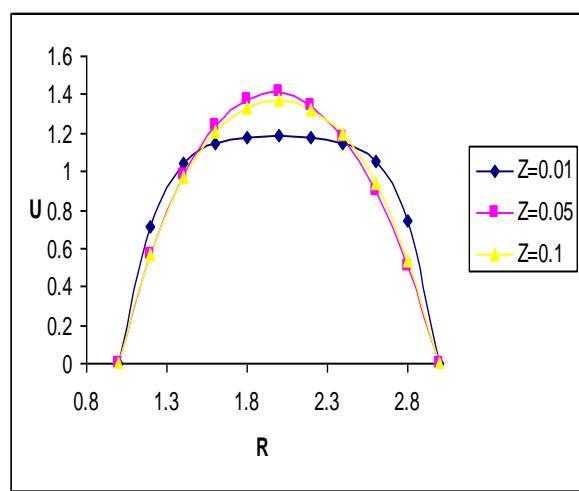

Fig.9: Velocity profile for fixed $M=0$ 


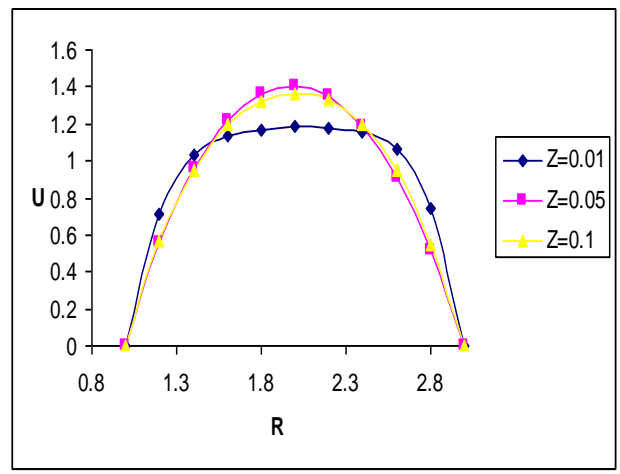

Fig.10: Velocity profile for $\mathrm{M}=2$

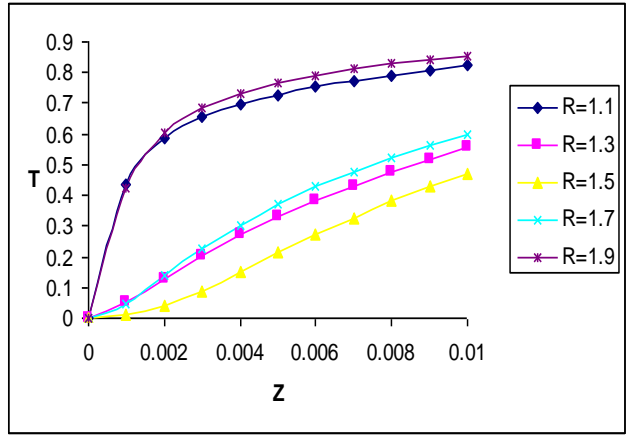

Fig.12: Temperature profile for fixed $\mathrm{M}=0$

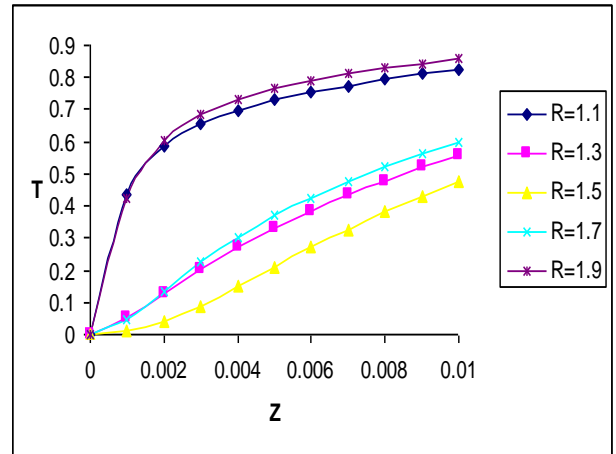

Fig.14: Temperature profile for fixed $M=4$

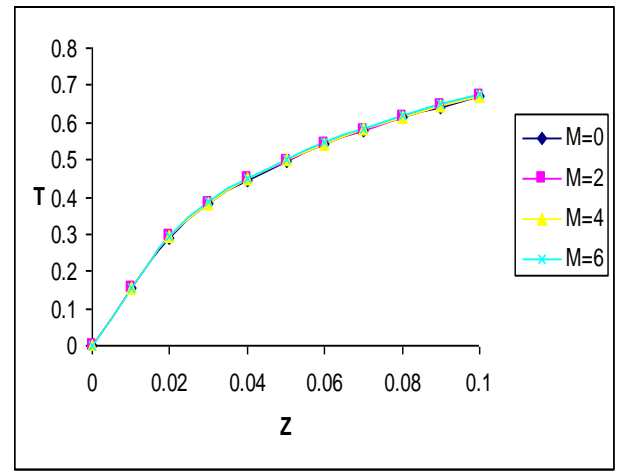

Fig.16: Temperature profile for fixed $\mathrm{R}=1.2$

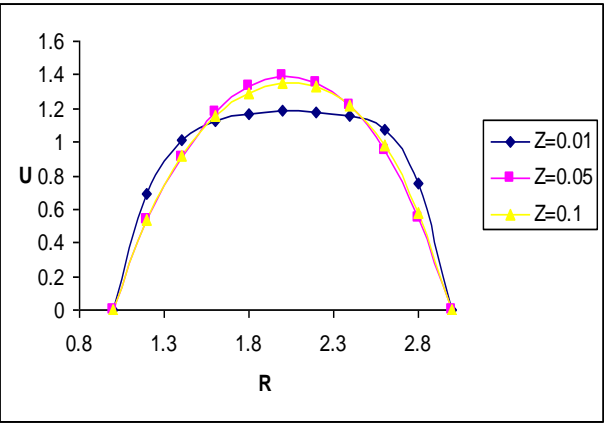

Fig.11: Velocity profile for fixed $M=4$

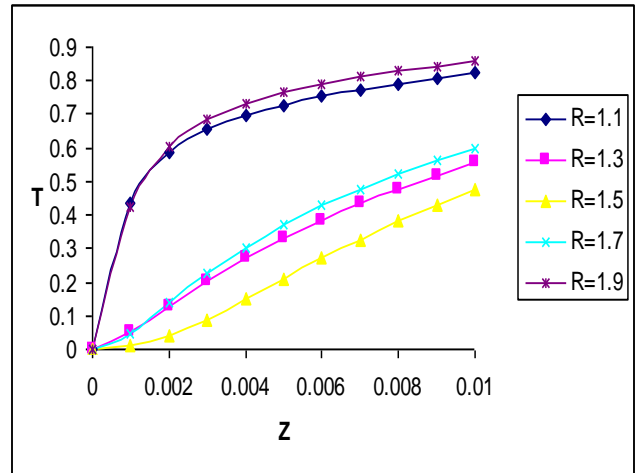

Fig.13: Temperature profile for fixed $M=2$

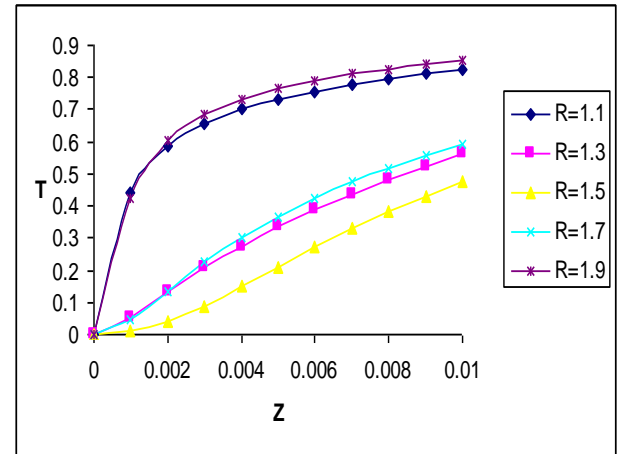

Fig.15: Temperature profile for fixed $\mathrm{M}=6$

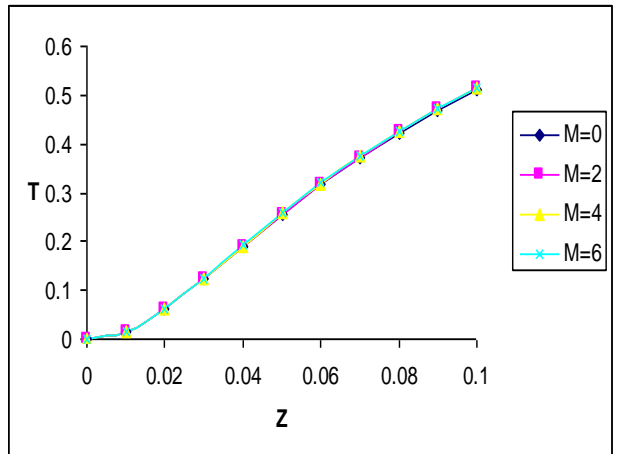

Fig.17: Temperature profile for fixed $\mathrm{R}=1.6$ 


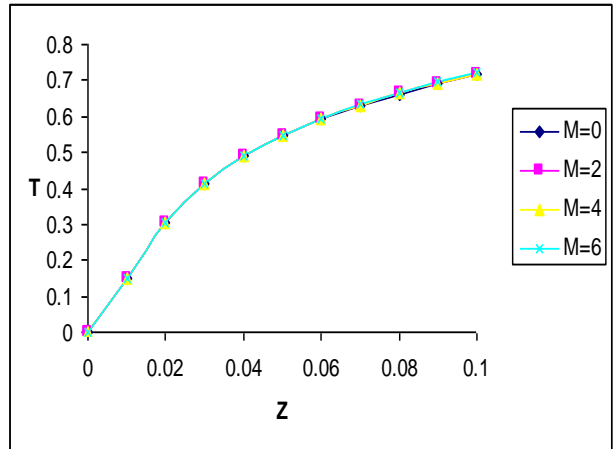

Fig.18: Temperature profile for fixed $\mathrm{R}=1.8$

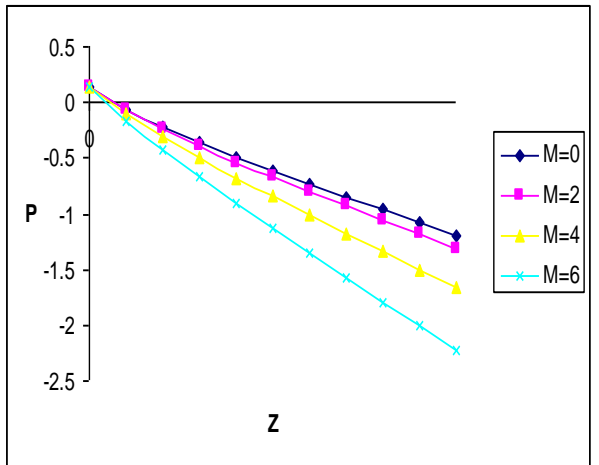

Fig. 20: Pressure profile for fixed $\mathrm{Z}=0.01$

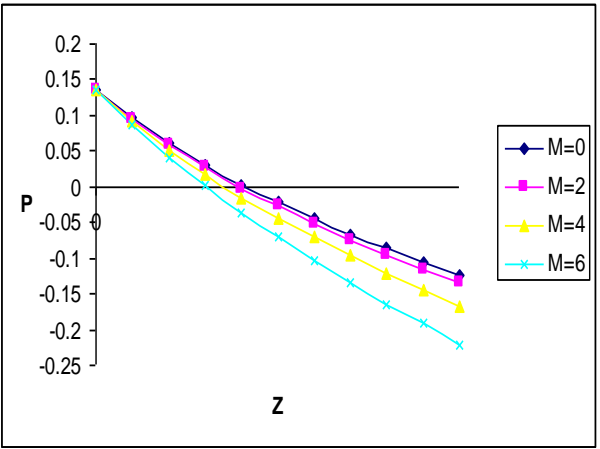

Fig. 19: Pressure profile for fixed $\mathrm{Z}=0.001$

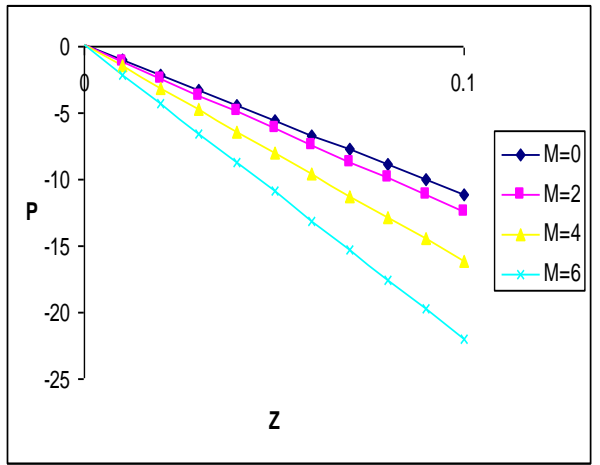

Fig.. 21: Pressure profile for fixed $\mathrm{Z}=0.1$

\section{References}

[1] Abdul Malaque, K.H and Abdul Sattar, Md., International Journal of Heat and Mass Transfer, Vol.48, p.4963, 2005.

[2] Andersson, H.I., and Korte. E.de., European Journal of Mechanics, Vol.21, p.317, 2002.

[3] Chekmarer, I.G. Zhur. Tekh fig.30, 601, 1960

[4] Globe, S. Phy. Fluids 2, 404, 1959.

[5] Hartmann, J. and Iazarus, F.Kgl. Danske Videnskab, Selekab, Matt-Phys. Medd.15 No.6 and 7, 1937.

[6] Ingham D.B. Journal of Aerosols Science, Vol.22, p.253, 1991.

[7] Jain, P.K., Nourmohammadi, K., and R.Y. R.P., Nuclear Engineering and design, Vol.60, p.401, 1980.

[8] Kumari, M., and Nath. G., International Journal of engineering science, Vol.42, p.1817, 2004

[9] Rossow, V.J., NACA - TN 3971, 1957.

[10] Shah. V.L., and Farnia, K., Computers and Fluids, Vol.2, p.285, 1974.

[11] Shohet, J.L. Osterte, J.F and Young, F.J. Phys. Fluids 5, 545, 1962.

[12] Sparrow, E.M., and Cess, R.D., Trans. ASME J. Appl. Mech. Vol.29, No.1, p.181, 1962

[13] Uflyand, Ya. Zhur.S. Tekh fig.30, 799, 1960. 